\title{
Self-reported adherence supports patient preference for the single tablet regimen (STR) in the current cART era
}

This article was published in the following Dove Press journal:

Patient Preference and Adherence

6 June 2012

Number of times this article has been viewed

\section{Gaetana Sterrantino' \\ Lucia Santoro' \\ Dario Bartolozzi \\ Michele Trotta' \\ Mauro Zaccarelli²}

'SOD Malattie Infettive, Azienda Ospedaliera Universitaria Careggi, Firenze, ${ }^{2}$ Istituto Nazionale per le Malattie Infettive "Lazzaro Spallanzani,” Roma, Italy
Correspondence: Gaetana Sterrantino SOD Malattie Infettive, Azienda Ospedaliera Universitaria Careggi, Largo Brambilla 3,

50134 Firenze, Italy

Tel +39055 7949242

$\mathrm{Fax}+390557949422$

Email sterrantinok@gmail.com
Objective: To analyze self-reported adherence to antiretroviral regimens containing ritonavirboosted protease inhibitors, nonnucleoside reverse transcriptase inhibitors (NNRTI), raltegravir, and maraviroc.

Methods: Overall, 372 consecutive subjects attending a reference center for HIV treatment in Florence, Italy, were enrolled in the study, from December 2010 to January 2012 (mean age 48 years). A self-report questionnaire was filled in. Patients were defined as "nonadherent" if reporting one of the following criteria: $<90 \%$ of pills taken in the last month, $\geq 1$ missed dose in the last week, spontaneous treatment interruptions reported, or refill problems in the last 3 months. Gender, age, CD4, HIV-RNA, years of therapy, and type of antiretroviral regimen were analyzed with respect to adherence.

Results: At the time of the questionnaire, $89.8 \%$ of patients had $<50$ copies/mL HIV-RNA and $14.2 \%$ were on their first combined antiretroviral therapy. $57 \%$ of patients were prescribed a regimen containing ritonavir boosted protease inhibitors (boosted PI), 41.7\% NNRTI, 17.2\% raltegravir, and $4.8 \%$ maraviroc; $49.5 \%$ of the subjects were on bis-in-die regimens, while $50.5 \%$ were on OD regimens, with $23.1 \%$ of these on the single tablet regimen (STR): tenofovir/ emtricitabine/efavirenz. The nonadherence proportion was lower in NNRTI than in boosted-PI treatments (19.4\% vs 30.2\%), and even lower in STR patients (17.4\%). In multivariable logistic regression, patients with the NNRTI regimen (OR: 0.56, 95\% CI: 0.34-0.94) and the STR (OR: 0.45, 95\% CI: 0.22-0.92) reported lower nonadherence. Efavirenz regimens were also associated with lower nonadherence (OR: 0.42, 95\% CI: 0.21-0.83), while atazanavir/ritonavir regimens were associated with higher nonadherence. No other relation to specific antiretroviral drugs was found. A higher CD4 count, lower HIV-RNA, and older age were also found to be associated with lower nonadherence, while a longer time on combined antiretroviral therapy was related to higher nonadherence.

Conclusion: STR maintains an advantage in improving adherence with respect to other combined antiretroviral therapies, even though new antiretroviral drugs and drug classes have become available in recent years.

Keywords: HIV, self-reported adherence, single tablet regimen, boosted protease inhibitors, nonnucleoside reverse-transcriptase inhibitors

\section{Introduction}

Combined antiretroviral therapy (cART) has changed the prognosis of most patients infected with HIV by decreasing morbidity and mortality. ${ }^{1}$ However, establishing a strong adherence to cART regimens remains a challenge for most patients. ${ }^{2,3}$ In addition, a comparison of results among different adherence studies will inevitably be biased because of the discrepancy between the employed definitions of adherence. 
Complex regimens decrease adherence, ${ }^{4}$ and thus maintaining adherence above $95 \%$ was originally considered necessary to avoid virological failure, ${ }^{5}$ but most studies anticipated the use of ritonavir-boosted protease inhibitor (boosted PI) regimens in first-line therapy, or included a large number of patients in un-boosted PI regimens. ${ }^{6}$

Whether this high degree of adherence is necessary for regimens containing boosted PI and nonnucleoside reverse transcriptase inhibitors (NNRTI) is still being debated. ${ }^{7}$ Recent studies have shown that an adherence to boosted PI greater than $80 \%$ may be sufficient. ${ }^{8,9}$ NNRTI could require even lower adherence rates than boosted PI, ${ }^{8}$ but the risk of acquiring NNRTI resistance is higher than for boosted PI. ${ }^{6}$ Moreover, studies analyzing adherence in maraviroc and raltegravir regimens are lacking.

Given the expectation of improved adherence for recently released antiretroviral drugs, thanks to improved tolerability and better toxicity profiles, the goal was to measure the adherence rate through self-reported adherence to antiretroviral regimens, including boosted PI, NNRTI, maraviroc, and raltegravir, and comparing the adherence in patients treated with different medications (twice daily [BID]; once-daily [OD]; and the single tablet regimen [STR]).

\section{Methods}

The study was conducted at the HIV clinic of Careggi Hospital in Florence, Italy. A standard interview concerning a brief self-administered questionnaire ${ }^{10}$ was carried out at each medical visit, after signing informed consent, by consecutive HIV infected patients on stable cART, who accessed the site between December 2010 and January 2012. Only patients who had received at least 3 months of treatment with the current regimen were included in the study.

The following four measurements of adherence comprised the assessment: (1) the proportion of cART doses taken over the preceding month, as measured by a visual analog scale; (2) any missed doses over the past week; (3) spontaneous treatment interruption of two or more days in the past three months; and (4) a lack of drug refill after finishing the drug in the past 3 months. Therefore, a lack of just one correct behavior was defined as nonadherence, as follows: less than $90 \%$ of pills taken in the last month, one, or more than one missed dose in the last week, a spontaneous treatment interruption, or refill problems in the last 3 months.

All clinical data were obtained by consulting the records of the hospital, including the demographic data, CD4 T-cell count, HIV-RNA values, Centers for Disease Control and Prevention (CDC) stages of clinical assessment, and therapeutic history.
Viral load and CD4 cell counts, collected within 3 months of filling out the questionnaire, were considered. Patients with viral loads lower than 50 copies $/ \mathrm{mL}$, in two consecutive measurements, were considered virologically suppressed.

Descriptive results are presented as proportions, medians, ranges, or 95\% confidence intervals. Inferential statistics, for unadjusted assessment, used Fisher's exact test to analyze categorical variables and the ANOVA test to analyze the continuous variables. Moreover, for multivariable assessment, adjusted logistic regression was also used.

The multivariable models included nonadherence, as previously defined, as a dependent variable, as well as variables significantly associated with unadjusted analysis. Gender, age, CD4 cell count, $\log _{10}$ HIV-RNA, AIDS (CDC C3) diagnosis, and frequency of drug prescription (BID or OD) were all included as covariates, regardless of their significance in unadjusted analysis. Each antiretroviral drug, drug class, and STR was added to the adjusted analysis in order to test whether they were associated with nonadherence.

\section{Results}

Out of the 427 consecutive patients attending the center during the observation time, 372 (87.1\%) were enrolled in the study (55 patients did not complete the adherence questionnaire; $60 \%$ of these were women). About half of the patients refused to complete the questionnaire (26 patients) because they reported that they were busy, while $20 \%$ were worried about their privacy.

The general characteristics of the included patients are described in Table 1. As shown, about one fourth had a previous AIDS diagnosis. Patients were generally pre-treated; indeed, only $14.2 \%$ were on for their first antiretroviral regimen. At the time of the questionnaire date, most of them (about 90\%) had undetectable HIV-RNA ( $<50$ copies $/ \mathrm{mL}$ ).

The proportion of patients using each drug, drug class, and type of regimen is reported in Table 2 . As shown, $>90 \%$ of patients were in treatment with a cART regimen, including

Table I General characteristics of included patients

\begin{tabular}{|c|c|c|}
\hline & n (\%) & Median IQR \\
\hline Male gender & $287(77.2)$ & \\
\hline Age (years) & & $47.7(42.1-54.5)$ \\
\hline CDC C3 & $91(24.5)$ & \\
\hline $\mathrm{CD}^{+}$cell count (cells $\left./ \mu \mathrm{L}\right)$ & & $602(423.5-825)$ \\
\hline Undetectable $\log _{10}$ HIV-RNA & $334(89.8)$ & \\
\hline HCV-positive & $76(20.4)$ & \\
\hline First line treatment & $53(14.2)$ & \\
\hline Overall duration of ART (months) & & $123(55.2-173)$ \\
\hline Duration of current cART (months) & & $19.2(11.0-36.4)$ \\
\hline
\end{tabular}

Abbreviations: $C D C$, Center for Disease Control and Prevention; IQR, interquartile range; ART, antiretroviral therapy; $C A R T$, combined antiretroviral therapy. 
Table 2 Level of adherence according to the drugs or the type of regimen prescribed

\begin{tabular}{llll}
\hline & n (\%) & $\begin{array}{l}\text { Mean \% pills } \\
\text { taken in the } \\
\text { past month }\end{array}$ & $\begin{array}{l}\text { \% nonadherent } \\
\text { patients } \\
\text { (P value) }\end{array}$ \\
\hline Overall & $372(100)$ & 96.4 & 25.5 \\
NRTI backbone & $335(90.1)$ & 96.5 & 26.0 \\
Efavirenz & $94(25.3)$ & 97.8 & $17.0(P<0.029)$ \\
Nevirapine & $48(12.9)$ & 94.4 & 22.9 \\
Etravirine & $14(3.8)$ & 95.0 & 21.4 \\
Lopinavir & $53(14.2)$ & 96.8 & 34.0 \\
Atazanavir & $101(27.2)$ & 95.8 & 28.7 \\
$\quad$ Boosted & 81 & 95.3 & 32.1 \\
$\quad$ Unboosted & 20 & 98.0 & 15.0 \\
Darunavir & $42(11.3)$ & 95.7 & 31.0 \\
$\quad$ OD & 32 & 95.9 & 31.3 \\
BID & 10 & 95.0 & 30.0 \\
fAPV/r & $11(3.0)$ & 99.1 & 9.1 \\
Maraviroc & $18(4.8)$ & 96.1 & 16.7 \\
Raltegravir & $64(17.2)$ & 96.6 & 23.4 \\
PI class & $212(57.0)$ & 96.2 & $30.2(P<0.018)$ \\
NNRTI class & $155(41.7)$ & 96.5 & $19.4(P<0.021)$ \\
BID regimen & $184(49.5)$ & 96.2 & 26.1 \\
OD regimen & $188(50.5)$ & 96.6 & 25.0 \\
STR & $86(23.1)$ & 97.8 & $17.4(P<0.05)$ \\
\hline Ab &
\end{tabular}

Abbreviations: STR, single tablet regimen: tenofovir/emtricitabine/efavirenz; OD, once-daily; BID, bis-in-die; NNRTI, nonnucleoside reverse transcriptase inhibitor; $\mathrm{NRTI}$, nucleoside reverse transcriptase inhibitor; PI, protease inhibitor.

the NRTI backbone. As the third drug, boosted PI was the widest used, followed by NNRTI, integrase inhibitors (raltegravir), and CCR5 inhibitors (maraviroc). About half were on BID and half were on OD regimens. Less than one fourth were on a single tablet regimen: the co-formulation of tenofovir/emtricitabine/efavirenz in a single pill was the only one available at the time of the interview.
Table 2 shows, in the second column, the mean percentage of self-reported adherence in the last month before the interview (the first and main question of the questionnaire) by drug, drug-class, and type of medication (graphically summarized in Figures 1 and 2). Moreover, in the third column of Table 2 (and graphically summarized in Figures 3 and 4), the proportion of nonadherent patients is reported. The overall proportion of nonadherent patients was $25.5 \%$, and the proportion of patients who failed adherence according to the four adherence-defining questions is as follows: (1) less than $90 \%$ of pills taken in the last month: $8.1 \%$; (2) one, or more than one, missed dose in the past week: $12.4 \%$; (3) spontaneous treatment interruption in the past three months: $7.3 \%$; and (4) a lack of refill in the past 3 months: $8.1 \%$.

As shown, the largest percentage of pills taken in the previous month was reported by patients in treatment with efavirenz for NNRTI, with fos-amprenavir (though the number of patients in treatment with this drug was very low) and unboosted atazanavir for PI. STR had a high adherence percentage, as compared to the OD and BID regimens. Moreover, the PI-class and the NNRTI-class included in the regimen had a similar percentage of pills taken in the previous month, though with completely different results when considering nonadherent patients; with the Fisher exact test, the NNRTI class was found to be associated with lower nonadherence, while the PI class was associated with higher nonadherence.

Efavirenz was the only single drug associated with lower nonadherence; STR was also found to be associated with the Fisher exact test. Table 3 reports the multivariable association

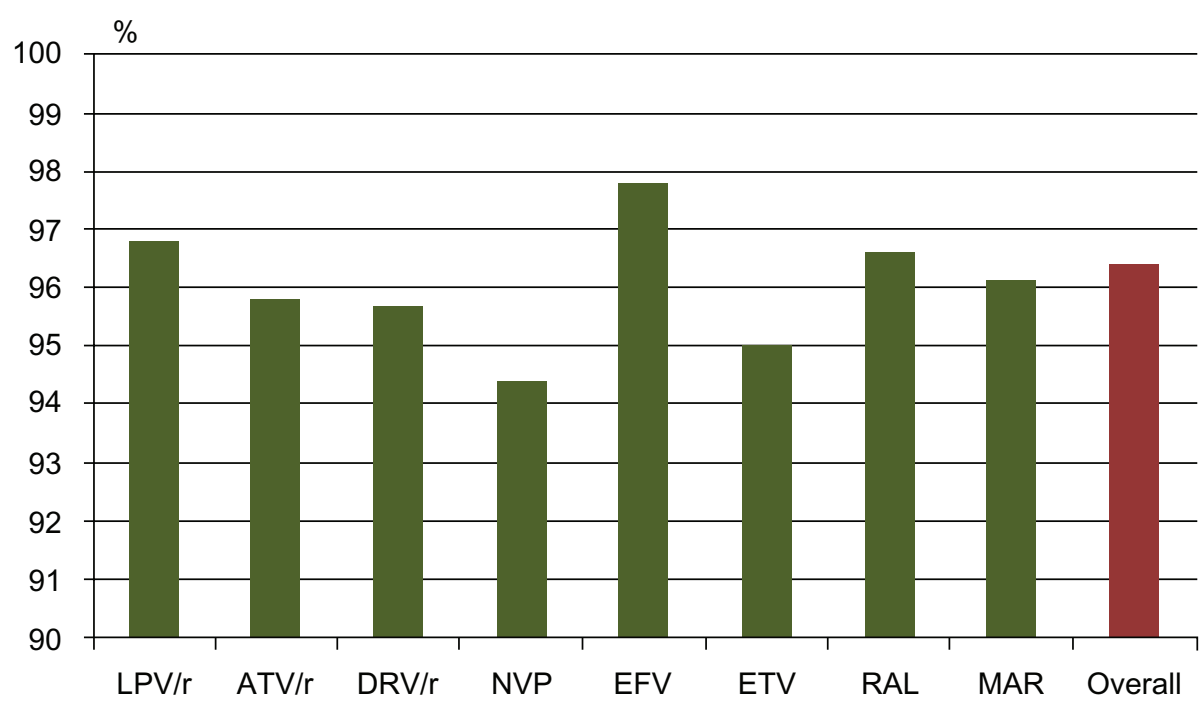

Figure I Self-reported proportion of pills taken in the previous month, according to the main drug used.

Abbreviations: LPV/r, Lopinavir/r; ATV/r, Atazanavir/ritonavir; DRV/r, Darunavir/ritonavir; NVP, Nevirapine; EFV, Efavirenz; ETV, Etravirine; RAL, Raltegravir; MVC, Maraviroc. 


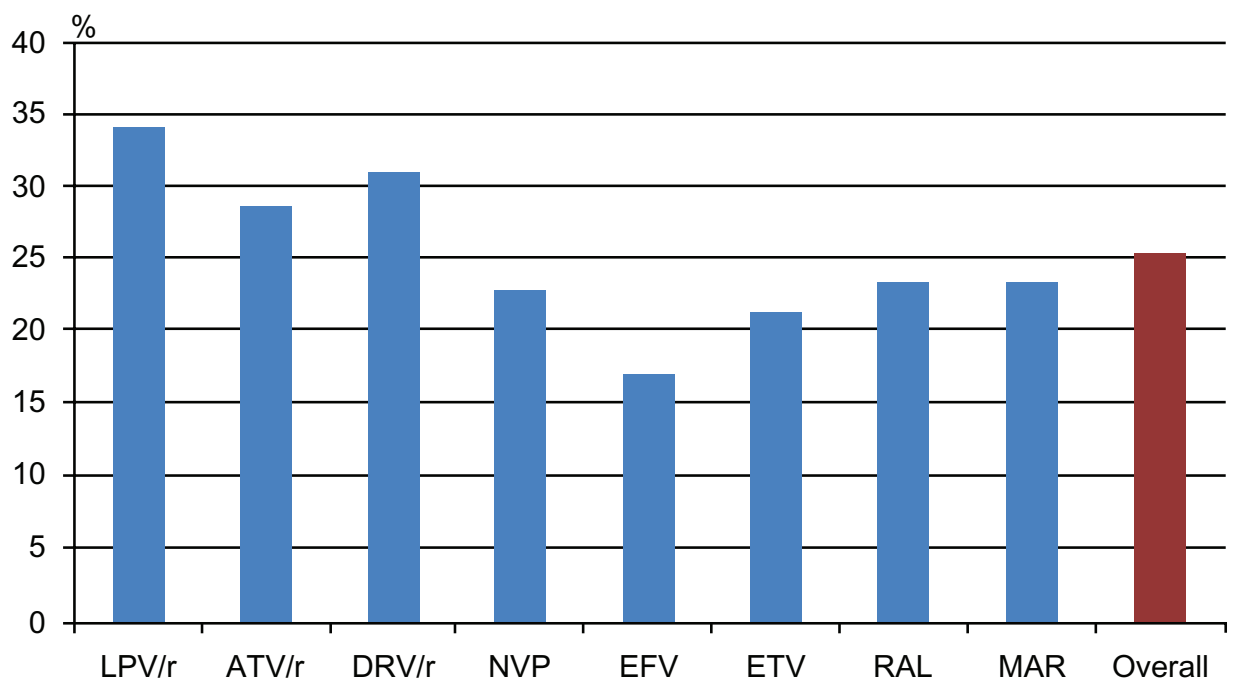

Figure 2 Proportion of patients reporting nonadherence, according to the main drug used (nonadherence defined as: $<90 \%$ pills taken in the past month; $\geq 1$ missed dose in the past week; a lack of drug refill in the previous 3 months; or a spontaneous drug interruption in the previous 3 months).

Abbreviations: LPV/r, Lopinavir/r; ATV/r, Atazanavir/ritonavir; DRV/r, Darunavir/ritonavir; NVP, Nevirapine; EFV, Efavirenz; ETV, Etravirine; RAL, Raltegravir; MVC, Maraviroc.

of single drugs with nonadherence. Efavirenz was associated with lower nonadherence, while boosted atazanavir was associated with higher nonadherence.

The other drugs were tested, but no significant association was observed (data not reported in the table). No association was found for the OD or BID regimens. Similarly, Table 4 reports the multivariable models for the NNRTI class and the STR, both of which are associated with lower nonadherence. The PI class in the current regimen was not found to be associated with nonadherence in the multivariable analysis.

Finally, in all models, a higher age and a higher CD4 cell count were associated with lower nonadherence, while a higher HIV-RNA and a longer time on cART were associated with higher nonadherence. HCV positivity, duration of current cART, and being on first line treatment were not associated with nonadherence in the unadjusted analysis, and therefore these were not included in the multivariable models.

\section{Discussion}

Monitoring and supporting adherence is of high importance for successful HIV treatment. ${ }^{11}$ Adherence was measured using a self-report questionnaire in an unselected sample of patients on steady cART; most of these patients (89.8\%)

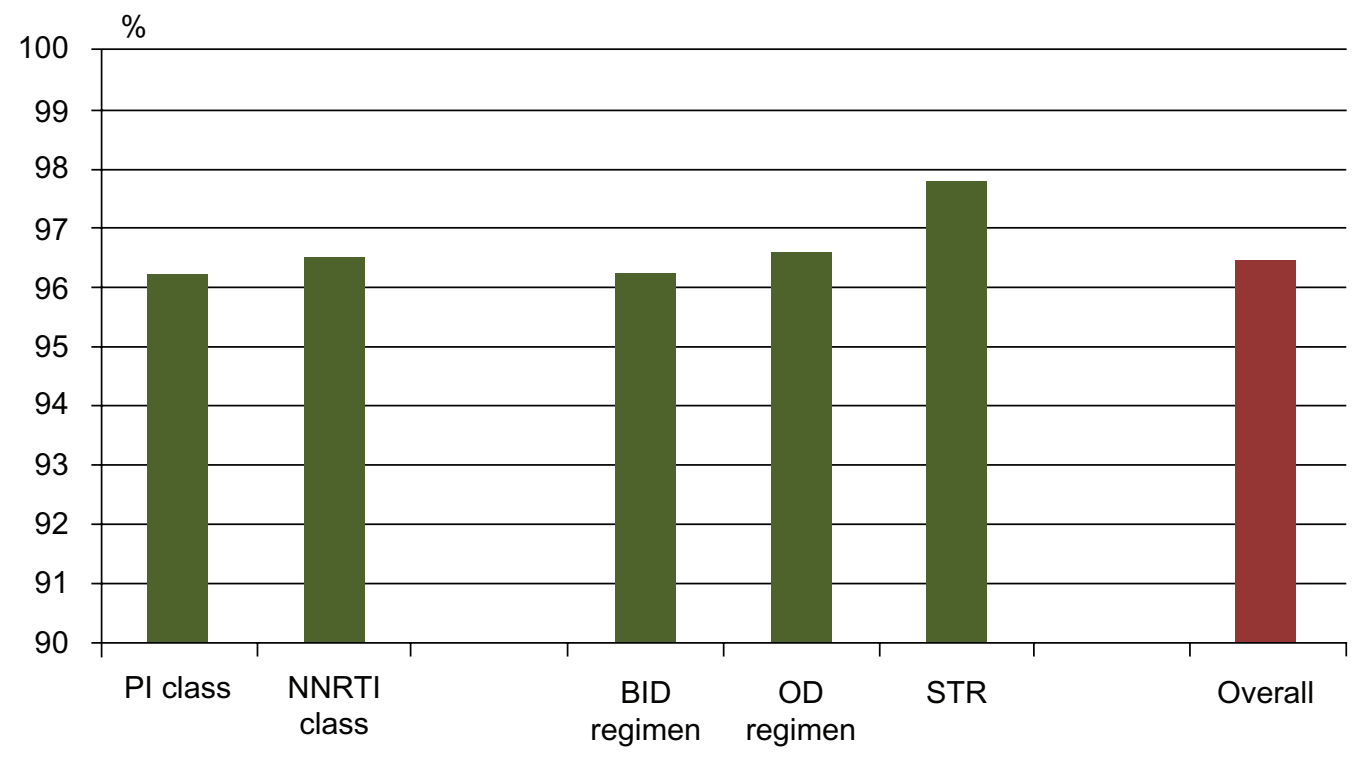

Figure 3 Self-reported proportion of pills taken in the last month, according to the type of regimen used.

Abbreviations: PI, protease inhibitor; NNRTI, nonnucleoside reverse transcriptase inhibitors; BID, twice daily; OD, once-daily; STR, single tablet regimen. 


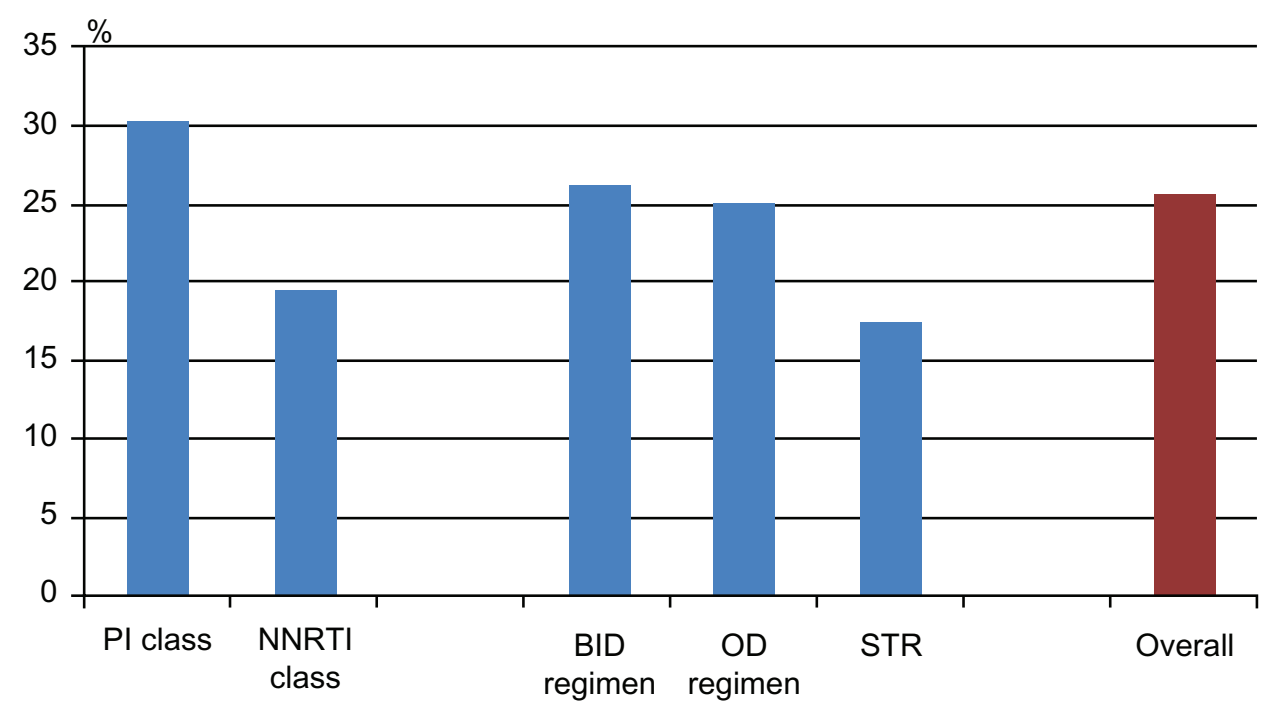

Figure 4 Proportion of patients reporting nonadherence, according to the type of regimen (nonadherence defined as: $<90 \%$ of pills taken in the previous month; $\geq \mathrm{I}$ missed dose in the previous week; a lack of drug refill in the previous 3 months; or a spontaneous drug interruption in the previous 3 months).

Abbreviations: PI, protease inhibitor; NNRTI, nonnucleoside reverse transcriptase inhibitor; BID, twice daily; OD, once-daily; STR, single tablet regimen.

were virologically suppressed (HIV-RNA $<50$ copies/mL) and/or in long-term treatment.

In our sample, the adherence level was high, which is possibly related to patients being in steady cART; moreover, self-reporting may overestimate the real level of adherence. ${ }^{1}$ However, relying on patients to report adherence is the easiest and most common method used for measurement.

In order to overcome the shortcomings of self-reported adherence, several questions were used to improve the robustness of the outcome: a "nonadherent" was defined as a subject who showed at least one nonadherence behavior, based on the the four questions included in the questionnaire.

After repeated testing, $90 \%$ of pills taken was chosen as the adherence cut-off point, derived from the assumption that with the recently released drugs (NNRTI, boosted PI, raltegravir, and maraviroc) this level would provide durable

Table 3 Adjusted logistic regression analysis for association with nonadherence in order to measure the effect of single drugs

\begin{tabular}{|c|c|c|}
\hline & $\begin{array}{l}\text { Efavirenz } \\
\text { OR }(95 \% \mathrm{Cl})\end{array}$ & $\begin{array}{l}\text { Boosted atazanavir } \\
\text { OR }(95 \% \mathrm{Cl})\end{array}$ \\
\hline Male gender & $0.88(0.48-1.60)$ & $0.89(0.49-1.60)$ \\
\hline Age (by year) & $0.96(0.93-0.99)$ & $0.96(0.94-0.99)$ \\
\hline $\mathrm{CDC} C 3$ & $1.17(0.66-2.06)$ & $1.17(0.67-2.07)$ \\
\hline CD4 (by $50 / \mu \mathrm{L}$ ) & $0.99(0.92-1.00)$ & $0.96(0.92-1.00)$ \\
\hline $\log _{10}$ HIV-RNA & $1.73(1.12-2.68)$ & $1.73(1.11-2.70)$ \\
\hline Years of therapy (each) & $1.07(1.02-1.12)$ & $1.07(1.02-1.12)$ \\
\hline OD regimen (vs BID) & $1.61(0.92-2.82)$ & $0.94(0.54-1.66)$ \\
\hline Efavirenz & $0.42(0.21-0.83)$ & \\
\hline Boosted atazanavir & & $1.88(1.01-3.54)$ \\
\hline
\end{tabular}

Abbreviations: OD, once-daily; BID, twice daily; CDC, Centers for Disease Control and Prevention. suppression. Indeed, recent reports have shown that durable viral suppression can be achieved using regimens that require adherence rates lower than $95 \% .^{12,13}$

No significant association was detected between nonadherence and gender. In contrast, a younger age was consistently associated with lower adherence to cART. ${ }^{14}$ Not surprisingly, a higher HIV-RNA and a lower CD4 count were associated with higher nonadherence, as was a longer time on cART, since (as previously observed) adherence may wane over time. ${ }^{15}$

The main finding of this analysis is related to the type of treatment. NNRTI treatment is associated with significantly lower nonadherence than boosted PI. In particular, efavirenz was associated with lower nonadherence, while boosted atazanavir was associated with higher nonadherence.

Table 4 Adjusted logistic regression analysis for association with nonadherence in order to measure the effect of single drug classes

\begin{tabular}{|c|c|c|}
\hline & $\begin{array}{l}\text { NNRTI } \\
\text { OR }(95 \% \mathrm{Cl})\end{array}$ & $\begin{array}{l}\text { STR } \\
\text { OR }(95 \% \mathrm{Cl})\end{array}$ \\
\hline Male gender & $0.90(0.50-1.64)$ & $0.88(0.47-1.60)$ \\
\hline Age (by year) & $0.96(0.94-0.99)$ & $0.96(0.93-0.99)$ \\
\hline CDC C3 & $1.16(0.67-2.04)$ & I. I $4(0.65-2.0 \mathrm{I})$ \\
\hline CD4 (by $50 / \mu \mathrm{L}$ ) & $0,96(0.92-1.00)$ & $0.96(0.92-1.00)$ \\
\hline $\log _{10}$ HIV-RNA & $1.66(1.08-2.57)$ & $1.69(1.09-2.61)$ \\
\hline Years of therapy (each) & $1.07(1.02-1.12)$ & $1.07(1.02-1.12)$ \\
\hline OD regimen (vs BID) & $1.23(0.74-2.05)$ & $1.60(0.9 \mathrm{I}-2.82)$ \\
\hline NNRTI Class & $0.56(0.34-0.94)$ & \\
\hline STR & & $0.45(0.22-0.42)$ \\
\hline
\end{tabular}

Abbreviations: STR, single tablet regimen: tenofovir/emtricitabine/efavirenz; OD, once-daily; BID, twice daily; NNRTI, nonnucleoside reverse transcriptase inhibitor; CDC, Centers for Disease Control and Prevention. 
However, the only available STR included efavirenz, so the improvement in adherence may be influenced more by STR than efavirenz. No significant differences were found when comparing the levels of adherence in patients on an OD regimen and a BID regimen. This partially contrasts with the results of a published meta-analysis, where adherence was modestly better with the OD regimen than with the BID regimen; this effect was more pronounced at the initiation of treatment and for regimens in which all medications were taken once per day. ${ }^{16}$

Our differing results could have several causes. First, most of the patients in this study were on long-term steady treatment, and thus as a result the study may include more adherent patients. Second, the BID regimens in our sample included raltegravir and maraviroc, which were found to be related to good, though nonsignificant, adherence levels.

Although data about symptoms were not collected, our data suggests that drugs with less toxicity, ie, still raltegravir or maraviroc used to switch from OD to BID regimen, may not result in adherence problems or loss of virological efficacy.

According to previous studies, simplifying the regimen to STR improved the adherence to the cART, ${ }^{17}$ and even switching from the OD regimen to STR, using the same drug combination, led to better reported adherence and quality of life. ${ }^{18}$ Our analysis appears to confirm these results, with one strong point being that the BID, OD, and STR regimens were compared in an actual treatment scenario in which new and more tolerable drugs, such as darunavir, etravirine, maraviroc, and raltegravir, were available.

The study has several limitations. Analysis is based on cross-sectional rather than prospective data, and therefore no causal conclusions can be drawn. The majority of the patients were on steady cART, and proportion of nonadherence may differ in patients starting cART de novo. The selection might have excluded those who experienced early virological failure, disease progression, or defaulted from care.

In conclusion, older age, higher CD4 cell counts, lower HIV-RNA viral loads, and the use of STR are all related to lower nonadherence. In particular, the use of STR maintains an advantage in improving adherence with respect to other cARTs, even with the availability of new, well-tolerated antiretroviral drugs and drug classes in recent years.

\section{Acknowledgments}

I wish to thank the patients who joined the study, as well as the medical staff of my department - Silvia Ambu, Beatrice
Borchi, Antonio Carocci, Paola Corsi, Alberto Farese, Canio Martinelli, Marcello Mazzetti, Marco Pozzi, and Pier Giorgio Rogasi - for helping to collect the adherence questionnaire.

\section{Disclosure}

I certify that that there are no actual or potential conflicts of interest in relation to this article.

\section{References}

1. Palella FJ Jr, Delaney KM, Moorman AC, et al. Declining morbidity and mortality among patients with advanced human immunodeficiency virus infection. N Engl J Med. 1998;338:853-860.

2. Bangsberg DR, Perry S, Charlebois ED, et al. Non-adherence to highly active antiretroviral therapy predicts progression to AIDS. AIDS. 2001; 15:1181-1183.

3. Garcia de Olalla P, Knobel H, Carmona A, Guelar A, López-Colomés JL, Caylà JA. Impact of adherence and highly active antiretroviral antiretroviral therapy on survival in HIV- infected patients. J Acquir Immune Defic Syndr. 2002;30:105-110.

4. Glass TR, De Geest S, Weber R, et al. Swiss HIV cohort study correlates of self-reported non-adherence to antiretroviral therapy in HIV-infected patients: the Swiss HIV Cohort Study. J Acquir Immune Defic Syndr. 2006;41:385-392.

5. Paterson DL, Swindells S, Mohr J, et al. Adherence to protease inhibitor therapy and outcomes in patients with HIV infection. Ann Intern Med. 2000;133:21-30.

6. Bangsberg DR, Acosta EP, Gupta R, et al. Adherence-resistance relationships for protease and non-nucleoside reverse transcriptase inhibitors explained by virological fitness. AIDS. 2006;20: 223-231.

7. Shuter J. Forgiveness of non-adherence to HIV-1 antiretroviral therapy. J Antimicrob Chemother. 2008;61:769-773.

8. Maggiolo F, Airoldi M, Kleinloog HD, et al. Effect of adherence to HAART on virologic outcome and on the selection of resistance-conferring mutations in NNRTI- or PI-treated patients. HIV Clin Trials. 2007; 8:282-292.

9. Parienti JJ, Ragland K, Lucht F, et al. Average adherence to boosted protease inhibitor therapy, rather than the pattern of missed doses, as a predictor of HIV RNA replication. Clin Infect Dis. 2010;50: 1192-1197.

10. Antinori A, Cozzi-Lepri A, Ammassari A, et al. Relative prognostic value of self-reported adherence and plasma NNRTI/PI concentrations to predict virological rebound in patients initially responding to HAART. Antivir Ther. 2004;9:291-296.

11. Bangsberg DR. A paradigm shift to prevent HIV drug resistance. PLoS Med. 2008;5(5):e111.

12. Maggiolo F, Ravasio L, Ripamonti D, et al. Similar adherence rates favour different virologic outcomes for patients treated with nonnucleoside analogues or protease inhibitors. Clin Infect Dis. 2005;40: $158-163$.

13. Bangsberg D. Less than $95 \%$ adherence to non-nucleoside reverse transcriptase inhibitor therapy can lead to viral suppression. Clin Infect Dis. 2006;43:939-941.

14. Fogarty L, Roter D, Larson S, Burke J, Gillespie J, Levy R. Patient adherence to HIV medication regimens: a review of published and abstract reports. Patient Educ Couns. 2002;46: 93-108.

15. Mannheimer S, Friedland G, Matts J, Child C, Chesney M. The consistency of adherence to antiretroviral therapy predicts biologic outcomes for human immunodeficiency virus-infected persons in clinical trials. Clin Infect Dis. 2002;34:1115-1121. 
16. Parienti JJ, Bangsberg DR, Verdon R, Gardner EM. Better adherence with once-daily antiretroviral regimens: a meta-analysis. Clin Infect Dis. 2009;48:484-488.

17. Dejesus E, Young B, Morales-Ramirez JO, et al. Simplification of antiretroviral therapy to a single-tablet regimen consisting of efavirenz, emtricitabine, and tenofovir disoproxil fumarate versus unmodified antiretroviral therapy in virologically suppressed HIV-1-infected patients. J Acquir Immune Defic Syndr. 2009;51:163-174.
18. Airoldi M, Zaccarelli M, Bisi L, et al. One-pill once-a-day HAART: a simplification strategy that improves adherence and quality of life of HIV-infected subjects. Patient Prefer Adherence. 2010;4:115-125.

\section{Publish your work in this journal}

Patient Preference and Adherence is an international, peer-reviewed, open access journal focusing on the growing importance of patient preference and adherence throughout the therapeutic continuum. Patient satisfaction, acceptability, quality of life, compliance, persistence and their role in developing new therapeutic modalities and compounds to optimize clinical outcomes for existing disease states are major areas of interest. This journal has been accepted for indexing on PubMed Central. The manuscript management system is completely online and includes a very quick and fair peer-review system. Visit http://www.dovepress.com/ testimonials.php to read real quotes from published authors.

Submit your manuscript here: http://www.dovepress.com/patient-preference-and-adherence-journal 